\title{
Hepatic steatosis does not cause insulin resistance in people with familial hypobetalipoproteinaemia
}

\author{
M. E. Visser • N. M. Lammers • A. J. Nederveen • \\ M. van der Graaf • A. Heerschap • M. T. Ackermans • \\ H. P. Sauerwein • E. S. Stroes • M. J. Serlie
}

Received: 31 December 2010 / Accepted: 28 March 2011/Published online: 6 May 2011

(C) The Author(s) 2011. This article is published with open access at Springerlink.com

\begin{abstract}
Aims/hypothesis Hepatic steatosis is strongly associated with hepatic and whole-body insulin resistance. It has proved difficult to determine whether hepatic steatosis itself is a direct cause of insulin resistance. In patients with familial hypobetalipoproteinaemia (FHBL), hepatic steatosis is a direct consequence of impaired hepatic VLDL excretion, independently of metabolic derangements. Thus, patients with FHBL provide a unique opportunity to investigate the relation between increased liver fat and insulin sensitivity.
\end{abstract}

Electronic supplementary material The online version of this article (doi:10.1007/s00125-011-2157-x) contains supplementary material, which is available to authorised users.

\section{E. Visser $\cdot$ E. S. Stroes $(\triangle)$}

Department of Vascular Medicine, Room F4.211,

Academic Medical Center,

Meibergdreef 9 ,

1105 AZ Amsterdam, the Netherlands

e-mail: E.S.Stroes@amc.uva.nl

\section{A. J. Nederveen \\ Department of Radiology, Academic Medical Center, \\ Amsterdam, the Netherlands}

N. M. Lammers $\cdot$ H. P. Sauerwein · M. J. Serlie

Department of Endocrinology and Metabolism,

Academic Medical Center,

Amsterdam, the Netherlands

\section{T. Ackermans}

Laboratory of Endocrinology, Academic Medical Center,

Amsterdam, the Netherlands

M. van der Graaf $\cdot$ A. Heerschap

Department of Radiology, Radboud University,

Nijmegen Medical Center,

Nijmegen, the Netherlands
Methods We included seven male participants with FHBL and seven healthy matched controls. Intrahepatic triacylglycerol content and intramyocellular lipid content were measured using localised proton magnetic resonance spectroscopy ('H-MRS). A two-step hyperinsulinaemiceuglycaemic clamp, using stable isotopes, was assessed to determine hepatic and peripheral insulin sensitivity.

Results ${ }^{1} \mathrm{H}-\mathrm{MRS}$ showed moderate to severe hepatic steatosis in patients with FHBL. Basal endogenous glucose production (EGP) and glucose levels did not differ between the two groups, whereas insulin levels tended to be higher in patients compared with controls. Insulin-mediated suppression of EGP during lower dose insulin infusion and insulin-mediated peripheral glucose uptake during higher dose insulin infusion were comparable between FHBL participants and controls. Baseline fatty acids and lipolysis (glycerol turnover) at baseline and during the clamp did not differ between groups. Conclusions/interpretation In spite of moderate to severe hepatic steatosis, people with FHBL do not display a reduction in hepatic or peripheral insulin sensitivity compared with healthy matched controls. These results indicate that hepatic steatosis per se is not a causal factor leading to insulin resistance.

\section{Trial registration: ISRCTN35161775}

Funding: This study was funded by the Departments of Vascular Medicine and Endocrinology and Metabolism of the Academic Medical Center Amsterdam.

Keywords Endogenous glucose production - Familial hypobetalipoproteinaemia $\cdot$ FHBL $\cdot$ Glucose disposal . Hepatic insulin resistance $\cdot$ Hepatic steatosis .

Hyperinsulinaemic-euglycaemic clamp - Insulin resistance . Non-alcoholic fatty liver disease $\cdot$ Peripheral insulin resistance 


$\begin{array}{ll}\text { Abbreviations } \\ \text { ApoB } & \text { Apolipoprotein B } \\ \text { CAT } & \text { Computed axial tomography } \\ \text { EGP } & \text { Endogenous glucose production } \\ \text { FFM } & \text { Fat-free mass } \\ \text { FHBL } & \text { Familial hypobetalipoproteinaemia } \\ \text { HOMA-IR } & \text { HOMA-insulin resistance } \\ { }^{1} \text { H-MRS } & \text { Proton magnetic resonance spectroscopy } \\ \text { IHTG } & \text { Intrahepatic triacylglycerol } \\ \text { IMCL } & \text { Intramyocellular lipid } \\ \text { NAFLD } & \text { Non-alcoholic fatty liver disease } \\ R_{\mathrm{d}} & \text { Rate of disappearance } \\ \text { REE } & \text { Resting energy expenditure }\end{array}$

\section{Introduction}

Non-alcoholic fatty liver disease (NAFLD) is a common feature of obesity and the metabolic syndrome $[1,2]$. NAFLD is the result of hepatic fat accumulation due to an increased flux of NEFA through the portal vein, reduced hepatic fatty acid oxidation and increased hepatic de novo lipogenesis, all of which are associated with central obesity and insulin resistance [3]. Hyperinsulinaemic-euglycaemic clamp studies have shown that increased intrahepatic triacylglycerol (IHTG) content strongly correlates with insulin resistance in liver, skeletal muscle and adipose tissue across a large range of liver fat percentages. Therefore, even small amounts of IHTG content were associated with metabolic dysfunction [1, 4-8]. In addition, results of animal studies have shown that hepatic fat accumulation may interfere with insulin signalling in the liver through activation of protein kinase $\mathrm{C} \varepsilon[9,10]$, suggesting a direct causal relationship between hepatic fat accumulation and insulin resistance. However, in obese humans it has been difficult to determine whether hepatic fat accumulation per se causes insulin resistance since both are features of metabolic derangements.

Familial hypobetalipoproteinaemia (FHBL) is a rare disorder of lipoprotein metabolism (estimated prevalence ranges from 1 in 500 to 1 in 1,000) and is characterised by LDL-cholesterol and total apolipoprotein B (ApoB) levels below the 5 th percentile $[11,12]$. Approximately $50 \%$ of FHBL patients are carriers of a mutation in the $A P O B$ gene [11] leading to the formation of a dysfunctional form of ApoB. Since ApoB is the main component of VLDL, mutations in $A P O B$ gene give rise to a defective VLDL export system with a reduced capacity to export triacylglycerol from the liver. As a consequence, mean triacylglycerol content in the livers of FHBL participants, measured using localised proton magnetic resonance spectroscopy $\left({ }^{1} \mathrm{H}-\right.$ MRS), is approximately three- to fivefold higher compared with that of controls [13]. Occasional reports on liver biopsies in FHBL patients have revealed moderate to severe steatosis, in some patients associated with mild inflammation and fibrosis $[11,14]$. However, hepatic triacylglycerol accumulation in FHBL, unlike in NAFLD, occurs predominantly independently of obesity-induced metabolic derangements [15]. For this reason FHBL patients provide a unique opportunity to investigate the relation between IHTG content and insulin sensitivity in humans.

In a previous study, hepatic steatosis in non-obese FHBL patients was associated with larger areas under the insulin curves of a $2 \mathrm{~h}$ glucose tolerance test when compared with healthy controls, although the difference was not statistically significant $[13,15]$. In another study, insulin sensitivity, assessed using the HOMA index, was similar in nonobese patients with FHBL compared with healthy controls [14]. More recently, Amaro et al. [16] showed that, during a hyperinsulinaemic-euglycaemic clamp, hepatic and peripheral insulin sensitivity did not differ between three obese participants with FHBL and six obese controls without hepatic steatosis.

In the present report we describe the results of an extensive study of glucose and fat metabolism in patients with FHBL. We performed a two-step hyperinsulinaemic-euglycaemic clamp with stable isotopes to determine hepatic and peripheral insulin sensitivity and total triacylglycerol lipolysis. In addition we measured intrahepatic triacylglycerol (IHTG) content and intramyocellular lipid (IMCL) content by magnetic resonance spectroscopy (MRS) and determined body fat distribution using dual energy X-ray absorptiometry and abdominal computed axial tomography.

\section{Methods}

Subjects We included seven male patients with documented FHBL and hepatic steatosis and seven healthy controls. We were not able to recruit more participants because of the low prevalence of FHBL and the strict inclusion criteria. Four of the FHBL patients had the same mutation in the $A P O B$ gene (11712delC). Two of these patients were brothers. The mutations identified in the other FHBL patients were 2534delA, Q1309X and 2783delC, respectively [17]. Patients and controls were matched for age, sex, BMI and WHR. Persons who performed regular exercise above sedentary level and those who were regularly using $>3$ units of alcohol per day or any recreational drug during the last 30 days were excluded from participation. Participants did not have any somatic illnesses, nor did they use supplements or medication influencing glucose or lipid metabolism. Oral glucose tolerance tests were within the normal range $(<7.8 \mathrm{mmol} / \mathrm{l})$ according to the criteria of the ADA [18]. All control participants had a normal routine blood examination. 
Written informed consent was obtained from all participants. The study protocol was approved by the local institutional review board. Patients were recruited from the outpatient clinic of the Academic Medical Center, Amsterdam, the Netherlands. Healthy volunteers were recruited via local advertisements.

Hyperinsulinaemic-euglycaemic clamp After an overnight fast, participants were admitted to the metabolic ward of the Academic Medical Center at 07:30 hours. Prior to the study day, all participants consumed at least $250 \mathrm{~g}$ of carbohydrates for 3 days and refrained from vigorous exercise for 1 week. A catheter was inserted into an antecubital vein for infusion of stable isotope tracers, insulin and glucose. Another catheter was inserted into a contralateral hand vein and kept in a thermoregulated $\left(60^{\circ} \mathrm{C}\right)$ Plexiglas box for sampling of arterialised venous blood. Saline was infused as $\mathrm{NaCl} 0.9 \%$ at a rate of $50 \mathrm{ml} / \mathrm{h}$ to sustain catheter patency. $\left[6,6-{ }^{2} \mathrm{H}_{2}\right]$ Glucose and $\left[1,1,2,3,3-{ }^{2} \mathrm{H}_{5}\right]$ glycerol were infused as tracers $(>99 \%$ enriched; Cambridge Isotopes, Andover, MA, USA) to study glucose kinetics and lipolysis (total triacylglycerol hydrolysis), respectively.

At time 0 (08:00 hours) blood samples were drawn for determination of background enrichments. A primed continuous infusion of isotopes was then started $\left(\left[6,6-{ }^{2} \mathrm{H}_{2}\right]-\right.$ glucose and $\left[1,1,2,3,3-{ }^{2} \mathrm{H}_{5}\right]$ glycerol, both at a rate of $0.11 \mu \mathrm{mol} \mathrm{kg} \mathrm{kmi}^{-1}$, with a priming dose equivalent to 80 min of infusion) and continued until the end of the study. After an equilibration time of $2.5 \mathrm{~h}$, three blood samples were taken for the measurement of isotope enrichments and one for the measurement of glucoregulatory hormones and NEFA. Thereafter, a two-step hyperinsulinaemic-euglycaemic clamp was started. A continuous infusion of insulin (Actrapid $100 \mathrm{U} / \mathrm{ml}$; Novo Nordisk Farma, Alphen aan de Rijn, the Netherlands) was started for $2 \mathrm{~h}$ and $10 \mathrm{~min}$ at the rate of $20 \mathrm{mU}\left[\mathrm{m}^{2} \text { body surface area }\right]^{-1} \mathrm{~min}^{-1}$, followed by an infusion of insulin at a rate of $60 \mathrm{mU}\left[\mathrm{m}^{2}\right.$ body surface area $]^{-1} \min ^{-1}$ for another $2 \mathrm{~h}$ and $10 \mathrm{~min}$. Plasma glucose levels were measured every $5 \mathrm{~min}$ at the bedside. Glucose was infused as $20 \%$ glucose at a variable rate, to maintain a plasma glucose concentration of $5.0 \mathrm{mmol} / \mathrm{l} .\left[6,6-{ }^{2} \mathrm{H}_{2}\right]$ Glucose was added to the $20 \%$ glucose solution to achieve glucose enrichments of $1 \%$ to approximate the values for enrichment reached in plasma and thereby minimise changes in isotopic enrichment due to changes in the infusion rate of exogenous glucose [19]. During the last $40 \mathrm{~min}$ of both hyperinsulinaemic periods, blood samples were drawn at $10 \mathrm{~min}$ intervals for determination of isotope enrichments, glucoregulatory hormones and NEFA. During the study day, participants remained fasted but were allowed to drink water.

Body composition and indirect calorimetry Body composition was measured by bioelectrical impedance analysis
(Maltron BF906; Maltron, Rayleigh, UK). Peripheral and trunk fat mass were quantified by dual-energy X-ray absorptiometry (QDR-4500W, software version whole body v8.26A: 5, Hologic, Bedford, MA, USA).

For determination of visceral and abdominal fat mass, a standardised single slice abdominal CAT-scan was performed through the level of the fourth lumbar vertebra (MX8000, Brilliance, Philips, Best, the Netherlands).

Oxygen consumption $\left(\dot{V} \mathrm{O}_{2}\right)$ and $\mathrm{CO}_{2}$ production $\left(\dot{V} \mathrm{CO}_{2}\right)$ were measured with the ventilated hood technique (model 2900; Sensormedics, Anaheim, CA, USA). $\dot{V} \mathrm{O}_{2}$ and $\dot{V} \mathrm{CO}_{2}$ were measured continuously during the final $30 \mathrm{~min}$ of the basal state and during the final $30 \mathrm{~min}$ of step 2 of the hyperinsulinaemic-euglycaemic clamp. The mean values of $\dot{V} \mathrm{O}_{2}$ and $\dot{V} \mathrm{CO}_{2}$ during the final 20 min were used for the calculation of glucose and fat oxidation.

In vivo ${ }^{1} H$-MRS IHTG content was measured by ${ }^{1} \mathrm{H}$-MRS. We hypothesised that the reduced delivery of VLDL triacylglycerols to peripheral tissues in FHBL might be associated with reduced storage of triacylglycerols in skeletal muscle. Therefore, we also measured intramyocellular lipid content in the soleus muscle. Measurements were performed after an overnight fast, within a 2 week time frame before or after the clamp test. For logistical reasons, scanning was performed at two separate sites with two different scanners. ${ }^{1} \mathrm{H}-\mathrm{MRS}$ spectra were acquired using a 3.0 T Magnetom Trio (Siemens, Erlangen, Germany) and a 3.0 T Intera (Philips, Best, the Netherlands). Identical scanning parameters were used in both situations. During the measurements, participants remained in the supine position within the MRI scanner.

For the measurement of IMCL content, the soleus muscle of the right leg was positioned within the homogeneous volume of the magnet. Scout images were acquired in order to position the volume of interest $15 \mathrm{~cm}$ beneath the tibia plateau. Two-dimensional chemical shift imaging MRS data were collected using a point-resolved spectroscopy sequence (PRESS) with the following parameters: repetition time (TR) $1,100 \mathrm{~ms}$, echo time (TE) $30 \mathrm{~ms}, 10 \mathrm{~mm}$ slice thickness, $32 \times 32$ matrix size, field of view $16 \times 16 \mathrm{~cm}$, acquisition time $13 \mathrm{~min}$, one acquisition. Spectra with and without water suppression were obtained. A number of voxels ranging from 5 to 27 inside the soleus were selected for further analysis and were processed using the freely available 3DiCSI package (version 1.9.11; Columbia University, New York, NY, USA). The number of voxels selected for analysis was similar between the two sites. Chemical shifts were reported using water as the internal standard at $4.65 \mathrm{ppm}$. Average spectra were then processed using specialised computer software (jMRUI 2.2) [20]. Three 
peaks were line-fitted: IMCL $\left(\mathrm{CH}_{2}\right)$ and extramyocellular lipid content $\left(\mathrm{CH}_{2}\right.$ and $\left.\mathrm{CH}_{3}\right)$ peaks. Lipid content was calculated from the peak areas of IMCL $\mathrm{CH}_{2}$ (methylene) at $1.3 \mathrm{ppm}$. IMCL contents were then expressed as the percentage of water content. In one FHBL patient quantification of IMCL content was impossible because of a large extramyocellular lipid content.

IHTG content was obtained using single-voxel ${ }^{1} \mathrm{H}-\mathrm{MRS}$, using a body array coil as the transmitter and phased surface coils as receivers. MRS measurements were acquired during breathhold, using single-voxel stimulated acquisition mode (TE/TR 20/3,000 ms, six acquisitions). Volumes of interest in the liver were located away from major vascular structures and bile ducts. Voxel size was $27 \mathrm{~mm}^{3}$. The water and fat resonance peaks, located at 4.65 and $1.3 \mathrm{ppm}$, were integrated using jMRUI software [20] and relative fat content was expressed as the ratio of the fat peak area over the cumulative water and fat peak areas. Calculated peak areas of water and fat were corrected for $\mathrm{T} 2$ relaxation ( $\mathrm{T} 2_{\text {water }}, 34 \mathrm{~ms} ; \mathrm{T} 2_{\text {fat }}, 68 \mathrm{~ms}$ ) [21] and the percentage hepatic fat content was calculated according to Szczepaniak et al. [22].

Glucose and lipid metabolism measurements Plasma glucose concentrations were measured with the glucose oxidase method using a Biosen C-line plus glucose analyser (EKF Diagnostics, Barbleben/Magdeburg, Germany). Plasma NEFA concentrations were determined with an enzymatic colorimetric method (NEFA-C test kit; Wako Chemicals, Neuss, Germany) with intra-assay variation of $1 \%$, interassay variation of $4-15 \%$ and a detection limit of $0.02 \mathrm{mmol} / 1 .\left[6,6{ }^{2} \mathrm{H}_{2}\right]$ Glucose enrichment (tracer-to-tracee ratio) was measured as described by Ackermans et al. [23]. $\left[6,6-{ }^{2} \mathrm{H}_{2}\right]$ Glucose enrichment intra-assay variation was $0.5-$ $1 \%$ with an inter-assay variation of $1 \%$ and a detection limit of $0.04 \%$. [1,1,2,3,3- $\left.{ }^{2} \mathrm{H}_{5}\right]$ Glycerol enrichment was determined as described earlier [24]. Intra-assay variation was $1-3 \%$ for glycerol and $4 \%$ for $\left[1,1,2,3,3-{ }^{2} \mathrm{H}_{5}\right]$ glycerol; inter-assay variation was $2-3 \%$ for glycerol and $7 \%$ for $\left[1,1,2,3,3-{ }^{2} \mathrm{H}_{5}\right]$ glycerol.

Glucoregulatory hormones Insulin and cortisol were determined on an Immulite 2000 system (Diagnostic Products, Los Angeles, CA, USA). Insulin was measured with a chemiluminescent immunometric assay with intra-assay variation of $3-6 \%$, inter-assay variation of $4-6 \%$ and detection limit of $15 \mathrm{pmol} / \mathrm{l}$. Cortisol was measured with a chemiluminescent immunoassay with intra-assay variation of $7-8 \%$, inter-assay variation of $7-8 \%$ and a detection limit of $50 \mathrm{nmol} / \mathrm{l}$. Glucagon was determined with the Linco 125I RIA (Linco Research, St Charles, MO, USA) with an intra-assay variation of $9-10 \%$, inter-assay variation of $5-7 \%$ and detection limit of $15 \mathrm{ng} / \mathrm{l}$.
Calculations and statistics HOMA of insulin resistance (HOMA-IR) was calculated using the formula described previously by Matthews et al. [25]. Endogenous glucose production (EGP) and peripheral glucose uptake (rate of disappearance $\left[R_{\mathrm{d}}\right]$ ) were calculated using the modified forms of the Steele equations as described previously [19, 26]. EGP and $R_{\mathrm{d}}$ were expressed as $\mu \mathrm{mol}(\mathrm{kg} \mathrm{FFM})^{-1} \mathrm{~min}^{-1}$ (FFM, fat-free mass). Insulin clearance was calculated as the rate of insulin infusion ( $\mathrm{mU}\left[\mathrm{m}^{2} \text { body surface area }\right]^{-1}$ $\min ^{-1}$ ) divided by the mean plasma insulin concentration during the clamp [27]. Total triacylglycerol hydrolysis/ lipolysis (glycerol turnover) was calculated using formulas for steady-state kinetics adapted for stable isotopes [24, 26] and was expressed as $\mu \mathrm{mol} \mathrm{kg} \mathrm{min}^{-1}$.

Resting energy expenditure (REE), glucose oxidation and fat oxidation rates were calculated from $\dot{V} \mathrm{O}_{2}$ and $\dot{V} \mathrm{CO}_{2}$ as reported previously [28]. Non-oxidative glucose disposal was calculated as the difference between total glucose disposal and glucose oxidation.

All data were analysed with non-parametric tests. Comparisons between groups were performed using the Mann-Whitney $U$ test. SPSS version 14.0.2 (SPSS, Chicago, IL, USA) was used for statistical analysis. Data are presented as median (minimum-maximum). In the Electronic supplementary material (ESM) data are also presented as mean \pm SEM.

\section{Results}

Study participants We included seven male FHBL participants and seven healthy male controls. All patients were matched to controls for age, BMI and WHR. The baseline characteristics of the participants are listed in Table 1 and ESM Table 1. As expected, there was a significant difference in plasma levels of ApoB, LDL-cholesterol and triacylglycerol. In addition, plasma levels of alanine aminotransferase and aspartate aminotransferase were above the reference value, of 45 and $40 \mathrm{U} / 1$ respectively, in some FHBL patients but not in controls. The HOMA index tended to be higher in FHBL patients compared with controls, although the difference was not statistically significant.

Body composition and ${ }^{1} H-M R S$ Participants with FHBL did not differ in body composition from their matched controls (Table 2 and ESM Table 2). ${ }^{1}$ H-MRS measurements showed moderate hepatic steatosis in all participants with FHBL and mild steatosis in two control patients. As expected, IHTG content was significantly higher in participants with FHBL (29.8\% [15.9-38.0\%]) compared with controls $(0.8 \%$ [0.012.4\%]) $(p<0.005$; Fig. 1a). One FHBL patient showed a substantially higher IMCL content compared with all other 
Table 1 Baseline characteristics of FHBL patients and healthy controls

Data are median (range)

\begin{tabular}{lccl}
\hline Characteristic & FHBL $(n=7)$ & Control $(n=7)$ & $p$ value \\
\hline Age (years) & $47(22-61)$ & $45(22-60)$ & NS \\
BMI $\left(\mathrm{kg} / \mathrm{m}^{2}\right)$ & $27(22-32)$ & $26(20-36)$ & $\mathrm{NS}$ \\
WHR $(\mathrm{cm})$ & $1.02(0.97-1.05)$ & $1.01(0.94-1.12)$ & $\mathrm{NS}$ \\
Total cholesterol $(\mathrm{mmol} / \mathrm{l})$ & $1.94(1.39-2.81)$ & $4.75(3.47-5.23)$ & $<0.01$ \\
Triacylglycerol $(\mathrm{mmol} / \mathrm{l})$ & $0.14(0.05-0.49)$ & $0.76(0.40-1.07)$ & $<0.01$ \\
ApoB $(\mathrm{g} / \mathrm{l})$ & $0.24(0.12-0.4)$ & $0.83(0.54-0.90)$ & $<0.01$ \\
HDL $(\mathrm{mmol} / \mathrm{l})$ & $1.19(0.7-1.53)$ & $1.3(0.79-1.83)$ & $\mathrm{NS}$ \\
HOMA index & $1.58(0.21-2.35)$ & $0.76(0.23-1.58)$ & $\mathrm{NS}$ \\
Aspartate aminotransferase $(\mathrm{U} / \mathrm{l})$ & $39(22-49)$ & $28(14-34)$ & $<0.05$ \\
Alanine aminotransferase $(\mathrm{U} / \mathrm{l})$ & $62(22-106)$ & $23(10-32)$ & 0.05 \\
C-reactive protein $(\mathrm{mg} / \mathrm{l})$ & $1.4(0.50-3.10)$ & $1.0(0.50-6.20)$ & $\mathrm{NS}$ \\
\hline
\end{tabular}

participants but median IMCL concentrations did not significantly differ between groups (patients 7.7\% [4.5$10.1 \%]$, controls $5.1 \%$ [3.3-21.4\%]; not significant) (Fig. 1b).

Glucose metabolism Data on glucose metabolism are summarised in Table 3 and ESM Table 3.

At baseline, plasma glucose was comparable between groups, whereas insulin levels tended to be higher in participants with FHBL compared with controls.

EGP at baseline was $11.5(10.1-13.2) \mu \mathrm{mol}(\mathrm{kg} \mathrm{FFM})^{-1}$ $\min ^{-1}$ in patients and $13.0(11.4-14.0) \mu \mathrm{mol}(\mathrm{kg} \mathrm{FFM})^{-1}$ $\min ^{-1}$ in controls $(p=0.3)$. Insulin infusion during the first step of the clamp procedure increased plasma insulin concentrations to 199 (177-302) pmol/1 in FHBL participants and to $162(132-294) \mathrm{pmol} / \mathrm{l}$ in healthy controls $(p=0.2)$. During the second step of the clamp procedure insulin concentrations increased to $615(572-836) \mathrm{pmol} / \mathrm{l}$ in FHBL and to 504 (426-712) pmol/1 in control participants $(p=0.1)$.

Plasma levels for other glucoregulatory hormones, e.g. cortisol and glucagon, were comparable between groups both in the basal state and during the clamp (data not shown).
The ability of insulin to suppress endogenous glucose production was not impaired in FHBL patients compared with controls. At lower levels, insulin-mediated suppression of EGP was $68.6 \%(59.3-82.8 \%)$ in patients and $72.9 \%$ $(56.8-86.9 \%)$ in controls ( $p=0.9$; Fig. 2a). During higher dose insulin infusion EGP was completely suppressed in both groups.

The ability of insulin to increase peripheral glucose disposal was also not significantly impaired in patients with FHBL compared with controls (Fig. 2b). Correction of $R_{\mathrm{d}}$ for plasma insulin did not have an effect on our results (data not shown). Insulin clearance did not differ significantly between patients and controls during either step of the clamp.

REE, the basal rate of glucose oxidation and the insulinstimulated increase in glucose oxidation and non-oxidative glucose disposal were similar between groups (Table 3 and ESM Table 3).

NEFA and lipolysis In the basal state, circulating concentrations of NEFAs were not different between patients and control participants (Table 3 and ESM Table 3). Likewise, lipolysis (glycerol turnover) and lipid oxidation were
Table 2 Body fat composition

Data are median (range)

\begin{tabular}{lccc}
\hline Variable & FHBL & Controls & $p$ value \\
\hline Body weight $(\mathrm{kg})$ & $86(70-110)$ & $87(71-114)$ & NS \\
Body fat $(\%)$ & $26(19-30)$ & $23(12-35)$ & NS \\
Lean body mass $(\mathrm{kg})$ & $61(49-77)$ & $64(58-67)$ & $\mathrm{NS}$ \\
Peripheral fat mass $(\mathrm{kg})$ & $8.4(5.7-14.1)$ & $8.6(3.9-16.6)$ & $\mathrm{NS}$ \\
Trunk fat mass $(\mathrm{kg})$ & $11.7(6.1-16.7)$ & $10.8(3.6-19.9)$ & $\mathrm{NS}$ \\
Abdomen & & & \\
Subcutaneous adipose tissue $\left(\mathrm{cm}^{2}\right)$ & $189.31(148.43-429.06)$ & $203.35(47.76-504.59)$ & $\mathrm{NS}$ \\
Visceral adipose tissue $\left(\mathrm{cm}^{2}\right)$ & $124.20(38.71-180.71)$ & $106.61(26.46-218.99)$ & $\mathrm{NS}$ \\
Visceral:subcutaneous adipose tissue ratio & $0.62(0.26-1.19)$ & $0.55(0.22-1.08)$ & $\mathrm{NS}$ \\
\hline
\end{tabular}



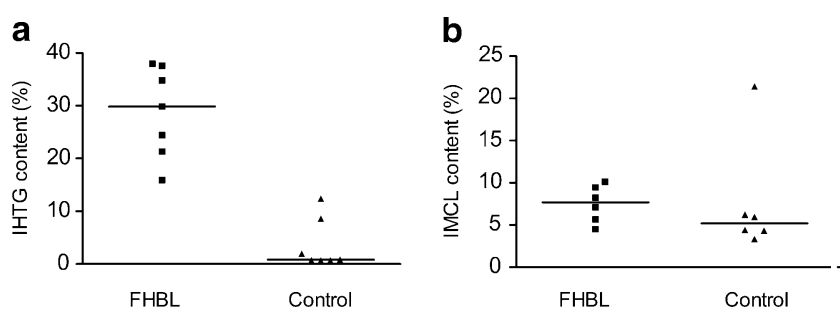

Fig. 1 IHTG and IMCL content assessed by MRS in participants with FHBL and healthy controls. The horizontal line represents the median. a Intrahepatic triacylglycerol (IHTG) content $(p<0.005)$. b Intramyocellular lipid content (IMCL) content (NS)

comparable between patients and controls both in the basal state and during the clamp.

Baseline lipolysis corrected for REE did not have an effect on the results (data not shown). Furthermore, lipolysis tended to be less suppressed by insulin in patients $60 \%(37-73 \%)$ compared with controls $66 \%$ $(55-75 \%)$, although the difference was not statistically significant ( $p=0.09$; Fig. $2 \mathrm{c}$ ).

\section{Discussion}

In the present study we show that patients with FHBL, despite the presence of moderate to severe hepatic steatosis, do not display a decrease in hepatic or peripheral insulin sensitivity compared with unaffected, matched controls. This indicates that hepatic triacylglycerol accumulation in itself is not causally related to hepatic or peripheral insulin resistance.

In the basal state, HOMA-IR tended to be higher in FHBL patients compared with controls. Since glucose levels were similar between patients and controls, the difference in HOMA-IR is probably explained by higher basal insulin levels in patients than in controls and is most likely caused by reduced insulin clearance and not by increased insulin secretion. This would be in line with earlier observations in non-diabetic participants, in whom hepatic fat accumulation was shown to be associated with impaired insulin clearance, independently of obesity [27]. A tendency towards higher insulin levels in FHBL patients was also observed during the hyperinsulinaemic clamp. Although calculation of insulin clearance during the clamp failed to show a significant difference between patients and controls, this finding further supports the concept that insulin clearance may be decreased in patients with FHBL.

It should be noted that when $R_{\mathrm{d}}$ was corrected for circulating plasma insulin levels, peripheral insulin sensitivity in FHBL patients did not differ from that in controls. Thus, despite a possible difference in insulin clearance, insulin- mediated peripheral glucose uptake was not impaired in patients with FHBL.

Two of the seven patients in the control group showed mild hepatic steatosis, of $12 \%$ and $8 \%$, respectively. One could argue that increased IHTG content in these patients might, to some extent, have skewed the data. However, since patients and controls were matched, individuals could not be excluded from analysis.

In a recent small study it was shown that both the hepatic insulin sensitivity index (inverse of the product of the basal EGP and fasting plasma insulin concentration) and the insulin-mediated increase in $R_{\mathrm{d}}$ did not differ between three obese participants with FHBL and six obese controls without hepatic steatosis [16]. In that study only a highdose insulin infusion $\left(50 \mathrm{mU} / \mathrm{m}^{2}\right)$ was used during the clamp. In the present study we extended these findings in a larger number of patients, including both lean and obese FHBL patients, and carefully matched controls. Moreover, by using both a high- and a low-dose insulin infusion we were able to unambiguously demonstrate that FHBL does not affect hepatic insulin sensitivity. In addition, we show that FHBL does not lead to impaired sensitivity in other target pathways of insulin, e.g. glucose oxidation, nonoxidative glucose disposal, lipolysis and lipid oxidation. Thus, our data provide strong evidence to show that in patients with FHBL, hepatic steatosis is a determinant of neither hepatic nor peripheral (muscle or adipose tissue) insulin resistance.

The lack of hepatic insulin resistance in FHBL in spite of severe steatosis may be surprising. Absence of insulin resistance has, however, been observed previously in animal models of hepatic steatosis [29]. In these animals, overabundance of hepatic diacylglycerol acyltransferase, an enzyme catalysing the final step in triacylglycerol synthesis [30]; deletion of long-chain fatty acid elongase family member 6 (ELOVL6), a microsomal enzyme involved in the elongation of fatty acids [31]; deletion of microsomal triacylglycerol transfer protein, responsible for the assembly of triacylglycerol-rich lipoproteins [32]; and pharmacological blockade of hepatic fatty acid $\beta$-oxidation [33], have all been associated with the induction of hepatic steatosis without hepatic or peripheral insulin resistance.

If hepatic steatosis in itself does not cause insulin resistance, factors other than hepatic triacylglycerol accumulation must be responsible for the close relation between these two entities in epidemiological studies [1, 4, 6-8]. Thus, hepatic steatosis and insulin resistance could represent two separate manifestations of the same metabolic derangements, such as chronic inflammation, endoplasmic reticulum stress or stress caused by other, as yet unidentified, metabolites [34, 35]. Recent studies have put forward the concept that lipid metabolites such as fatty acids, long- 


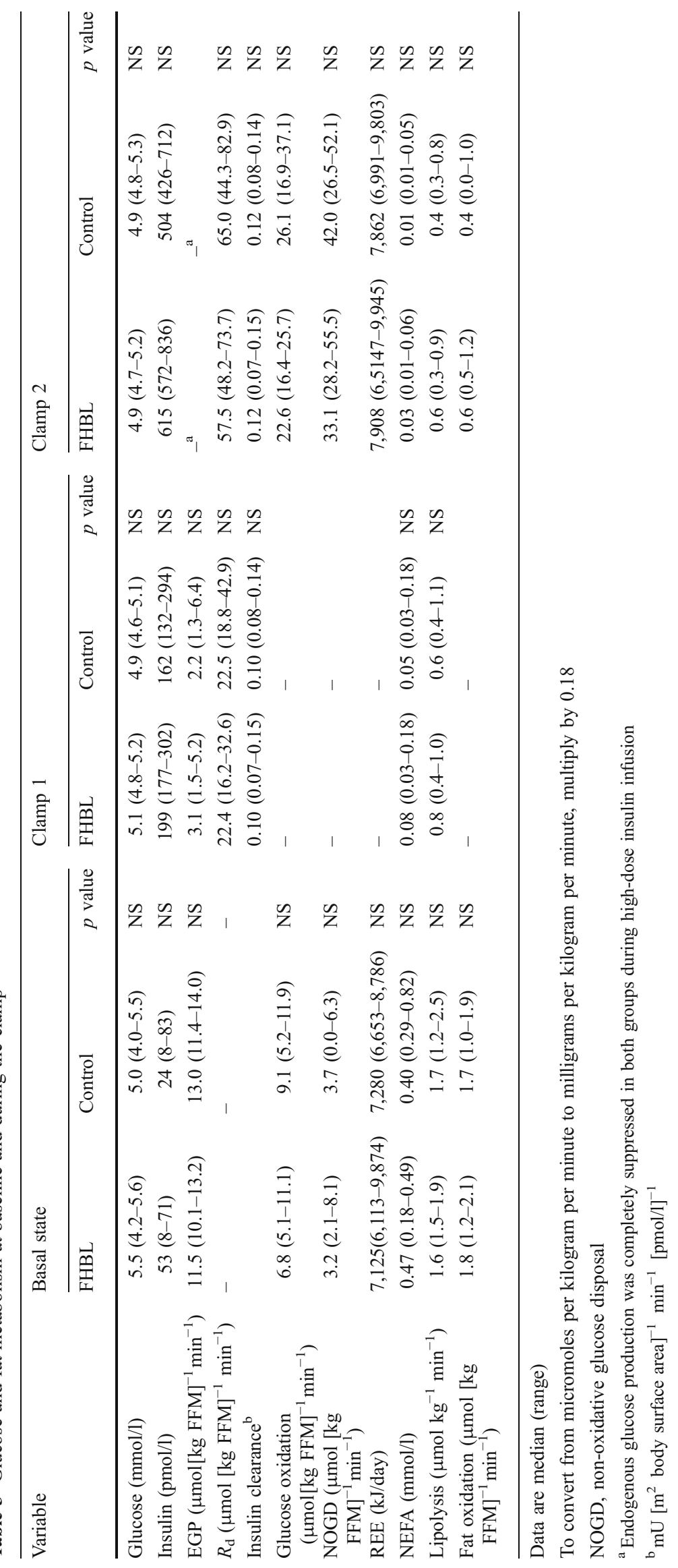



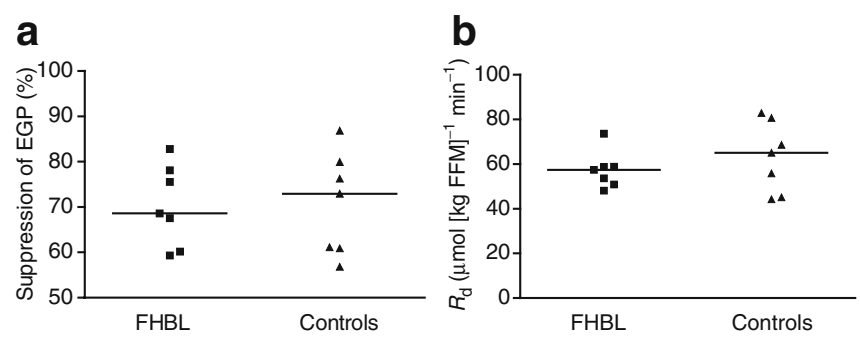

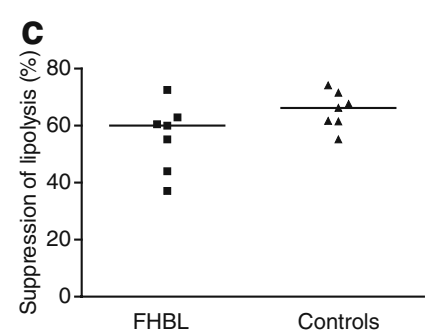

Fig. 2 Clamp results in participants with FHBL and healthy controls. The horizontal line represents the median. a Suppression of EGP during low-dose insulin infusion $(p=0.40)$. b $R_{\mathrm{d}}$ of glucose during

chain acyl-CoAs, diacylglycerol and ceramides rather than triacylglycerols themselves [36, 37] are determinants of the onset of insulin resistance [29, 38-40]. In this scenario, hepatic steatosis in FHBL may be the result of a harmless accumulation of triacylglycerols, whereas NAFLD is the result of an accumulation of toxic lipid metabolites leading to insulin resistance. In the present study, circulating concentrations of NEFA were similar in FHBL patients compared with controls.

Furthermore, the association between hepatic steatosis and insulin resistance may have a genetic basis. For example, polymorphisms in APOC3 have recently been shown to be associated with both NAFLD and insulin resistance, whereas the single-nucleotide polymorphism rs738409 in PNPLA3 was associated with increased liver fat but not with insulin resistance [41, 42]. Unfortunately, the results of the present study do not provide answers to explain the true mechanism underlying the relationship between hepatic steatosis and insulin resistance.

Hepatic steatosis has been suggested to be causally related to hepatic as well as peripheral insulin resistance. In the present study, we convincingly show in a unique human model of severe fatty liver disease that hepatic steatosis is not associated with hepatic or peripheral insulin resistance. Whereas the results of the present study do not unravel the exact mechanisms underlying the complex relationship between these two highly prevalent metabolic disorders, further studies focusing on the comparison of different hepatic steatosis models in large cohorts are required.

Acknowledgements The authors wish to thank S. M. van den Berg and R. D. Snoeks (Department of Radiology, Academic Medical Center, Amsterdam) for technical assistance in performing MRS, A. J. Borgers and H. Venema (Department of Endocrinology and Radiology, Academic Medical Center, Amsterdam) for assistance in computed tomography scan analysis, and A. Ruiter (Laboratory of Endocrinology, Academic Medical Center, Amsterdam) for assistance in laboratory analysis. Special thanks go to M. R. Soeters and R. M. Blumer (Department of Endocrinology, Academic Medical Center, Amsterdam) for performing the first clamps of this research project. high-dose insulin infusion ( $p=0.34)$. $\mathbf{c}$ Suppression of lipolysis during low-dose insulin infusion $(p=0.09)$

Duality of interest statement The authors declare that there is no duality of interest associated with this manuscript.

Open Access This article is distributed under the terms of the Creative Commons Attribution Noncommercial License which permits any noncommercial use, distribution, and reproduction in any medium, provided the original author(s) and source are credited.

\section{References}

1. Marchesini G, Brizi M, Bianchi G et al (2001) Nonalcoholic fatty liver disease: a feature of the metabolic syndrome. Diabetes 50:1844-1850

2. Yki-Jarvinen H (2010) Liver fat in the pathogenesis of insulin resistance and type 2 diabetes. Dig Dis 28:203-209

3. Donnelly KL, Smith CI, Schwarzenberg SJ, Jessurun J, Boldt MD, Parks EJ (2005) Sources of fatty acids stored in liver and secreted via lipoproteins in patients with nonalcoholic fatty liver disease. J Clin Invest 115:1343-1351

4. Deivanayagam S, Mohammed BS, Vitola BE et al (2008) Nonalcoholic fatty liver disease is associated with hepatic and skeletal muscle insulin resistance in overweight adolescents. Am J Clin Nutr 88:257-262

5. Korenblat KM, Fabbrini E, Mohammed BS, Klein S (2008) Liver, muscle, and adipose tissue insulin action is directly related to intrahepatic triglyceride content in obese subjects. Gastroenterology 134:1369-1375

6. Kotronen A, Juurinen L, Tiikkainen M, Vehkavaara S, YkiJarvinen H (2008) Increased liver fat, impaired insulin clearance, and hepatic and adipose tissue insulin resistance in type 2 diabetes. Gastroenterology 135:122-130

7. Seppala-Lindroos A, Vehkavaara S, Hakkinen AM et al (2002) Fat accumulation in the liver is associated with defects in insulin suppression of glucose production and serum free fatty acids independent of obesity in normal men. J Clin Endocrinol Metab 87:3023-3028

8. Staehr P, Hother-Nielsen O, Levin K, Holst JJ, Beck-Nielsen H (2001) Assessment of hepatic insulin action in obese type 2 diabetic patients. Diabetes 50:1363-1370

9. Samuel VT, Petersen KF, Shulman GI (2010) Lipid-induced insulin resistance: unravelling the mechanism. Lancet 375:2267-2277

10. Samuel VT, Liu ZX, Wang A et al (2007) Inhibition of protein kinase $\mathrm{C}$ epsilon prevents hepatic insulin resistance in nonalcoholic fatty liver disease. J Clin Invest 117:739-745

11. Tarugi P, Averna M, Di LE et al (2007) Molecular diagnosis of hypobetalipoproteinemia: an ENID review. Atherosclerosis 195: e19-e27 
12. Schonfeld G, Lin X, Yue P (2005) Familial hypobetalipoproteinemia: genetics and metabolism. Cell Mol Life Sci 62:1372-1378

13. Schonfeld G, Patterson BW, Yablonskiy DA et al (2003) Fatty liver in familial hypobetalipoproteinemia: triglyceride assembly into VLDL particles is affected by the extent of hepatic steatosis. J Lipid Res 44:470-478

14. Lonardo A, Lombardini S, Scaglioni F et al (2006) Hepatic steatosis and insulin resistance: does etiology make a difference? J Hepatol 44:190-196

15. Tanoli T, Yue P, Yablonskiy D, Schonfeld G (2004) Fatty liver in familial hypobetalipoproteinemia: roles of the APOB defects, intra-abdominal adipose tissue, and insulin sensitivity. J Lipid Res 45:941-947

16. Amaro A, Fabbrini E, Kars M et al (2010) Dissociation between intrahepatic triglyceride content and insulin resistance in familial hypobetalipoproteinemia. Gastroenterology 139:149-153

17. Fouchier SW, Sankatsing RR, Peter J et al (2005) High frequency of APOB gene mutations causing familial hypobetalipoproteinaemia in patients of Dutch and Spanish descent. J Med Genet 42:e23

18. American Diabetes Association (2010) Standards of medical care in diabetes-2010. Diabetes Care 33(Suppl 1):S11-S61

19. Finegood DT, Bergman RN, Vranic M (1987) Estimation of endogenous glucose production during hyperinsulinemic-euglycemic glucose clamps. Comparison of unlabeled and labeled exogenous glucose infusates. Diabetes 36:914-924

20. Naressi A, Couturier C, Devos JM et al (2001) Java-based graphical user interface for the MRUI quantitation package. MAGMA 12:141-152

21. de Bazelaire CM, Duhamel GD, Rofsky NM, Alsop DC (2004) MR imaging relaxation times of abdominal and pelvic tissues measured in vivo at 3.0 T: preliminary results. Radiology 230:652-659

22. Szczepaniak LS, Babcock EE, Schick F et al (1999) Measurement of intracellular triglyceride stores by $\mathrm{H}$ spectroscopy: validation in vivo. Am J Physiol 276:E977-E989

23. Ackermans MT, Pereira Arias AM, Bisschop PH, Endert E, Sauerwein HP, Romijn JA (2001) The quantification of gluconeogenesis in healthy men by $(2) \mathrm{H}_{2} \mathrm{O}$ and [2-(13)C]glycerol yields different results: rates of gluconeogenesis in healthy men measured with $(2) \mathrm{H}_{2} \mathrm{O}$ are higher than those measured with [2(13)C]glycerol. J Clin Endocrinol Metab 86:2220-2226

24. Ackermans MT, Ruiter AF, Endert E (1998) Determination of glycerol concentrations and glycerol isotopic enrichments in human plasma by gas chromatography/mass spectrometry. Anal Biochem 258:80-86

25. Matthews DR, Hosker JP, Rudenski AS, Naylor BA, Treacher DF, Turner RC (1985) Homeostasis model assessment: insulin resistance and beta-cell function from fasting plasma glucose and insulin concentrations in man. Diabetologia 28:412-419

26. Steele R (1959) Influences of glucose loading and of injected insulin on hepatic glucose output. Ann N Y Acad Sci 82:420-430
27. Kotronen A, Vehkavaara S, Seppala-Lindroos A, Bergholm R, Yki-Jarvinen H (2007) Effect of liver fat on insulin clearance. Am J Physiol Endocrinol Metab 293:E1709-E1715

28. Frayn KN (1983) Calculation of substrate oxidation rates in vivo from gaseous exchange. J Appl Physiol 55:628-634

29. Postic C, Girard J (2008) Contribution of de novo fatty acid synthesis to hepatic steatosis and insulin resistance: lessons from genetically engineered mice. J Clin Invest 118:829-838

30. Monetti M, Levin MC, Watt MJ et al (2007) Dissociation of hepatic steatosis and insulin resistance in mice overexpressing DGAT in the liver. Cell Metab 6:69-78

31. Matsuzaka T, Shimano H, Yahagi N et al (2007) Crucial role of a long-chain fatty acid elongase, Elovl6, in obesity-induced insulin resistance. Nat Med 13:1193-1202

32. Minehira K, Young SG, Villanueva CJ et al (2008) Blocking VLDL secretion causes hepatic steatosis but does not affect peripheral lipid stores or insulin sensitivity in mice. J Lipid Res 49:2038-2044

33. Grefhorst A, Hoekstra J, Derks TG et al (2005) Acute hepatic steatosis in mice by blocking beta-oxidation does not reduce insulin sensitivity of very-low-density lipoprotein production. Am J Physiol Gastrointest Liver Physiol 289:G592-G598

34. Arkan MC, Hevener AL, Greten FR et al (2005) IKK-beta links inflammation to obesity-induced insulin resistance. Nat Med 11:191-198

35. Shoelson SE, Herrero L, Naaz A (2007) Obesity, inflammation, and insulin resistance. Gastroenterology 132:2169-2180

36. Yamaguchi K, Yang L, McCall S et al (2007) Inhibiting triglyceride synthesis improves hepatic steatosis but exacerbates liver damage and fibrosis in obese mice with nonalcoholic steatohepatitis. Hepatology 45:1366-1374

37. Listenberger LL, Han X, Lewis SE et al (2003) Triglyceride accumulation protects against fatty acid-induced lipotoxicity. Proc Natl Acad Sci U S A 100:3077-3082

38. Savage DB, Petersen KF, Shulman GI (2007) Disordered lipid metabolism and the pathogenesis of insulin resistance. Physiol Rev 87:507-520

39. Wymann MP, Schneiter R (2008) Lipid signalling in disease. Nat Rev Mol Cell Biol 9:162-176

40. Li LO, Ellis JM, Paich HA et al (2009) Liver-specific loss of long chain acyl-CoA synthetase-1 decreases triacylglycerol synthesis and beta-oxidation and alters phospholipid fatty acid composition. J Biol Chem 284:27816-27826

41. Kantartzis K, Peter A, Machicao F et al (2009) Dissociation between fatty liver and insulin resistance in humans carrying a variant of the patatin-like phospholipase 3 gene. Diabetes 58:2616-2623

42. Petersen KF, Dufour S, Hariri A et al (2010) Apolipoprotein C3 gene variants in nonalcoholic fatty liver disease. N Engl J Med 362:1082-1089 